Documentation et bibliothèques

DOCUMENTATION BIBLIOTHËQUES

\title{
Les archives d'émissions de radio et de télévision au réseau français de la Société Radio-Canada
}

\section{Michelle Bachand}

Volume 35, numéro 1, janvier-mars 1989

URI : https://id.erudit.org/iderudit/1028122ar

DOI : https://doi.org/10.7202/1028122ar

Aller au sommaire du numéro

\section{Éditeur(s)}

Association pour l'avancement des sciences et des techniques de la documentation (ASTED)

\section{ISSN}

0315-2340 (imprimé)

2291-8949 (numérique)

Découvrir la revue

\section{Citer cet article}

Bachand, M. (1989). Les archives d'émissions de radio et de télévision au réseau français de la Société Radio-Canada. Documentation et bibliothèques, 35(1), 21-24. https://doi.org/10.7202/1028122ar

Tous droits réservés ( $)$ Association pour l'avancement des sciences et des techniques de la documentation (ASTED), 1989
Ce document est protégé par la loi sur le droit d'auteur. L'utilisation des services d'Érudit (y compris la reproduction) est assujettie à sa politique d'utilisation que vous pouvez consulter en ligne.

https://apropos.erudit.org/fr/usagers/politique-dutilisation/ 


\section{Les archives d'émissions de radio et de télévision au réseau français de la Société Radio-Canada}

Le centre de production du réseau français est situé à Montréal dans l'édifice connu sous le nom de Maison de Radio-Canada. II est sous la direction de deux vice-présidences: télévision française et radio française, lesquelles relèvent du siège social situé à Ottawa. Les archives d'émissions sont sous la responsabilité du secteur télévision, dans l'ordre hiérarchique suivant: la Direction des Approvisionnements et Services comprend le Service des ressources documentaires lequel chapeaute les archives d'émissions de radio et de télévision et la bibliothèque.

Les archives du réseau français ont commencé à se structurer en 1963 sous le nom d'archives de programmes relevant des Services généraux. Avec les années et les changements d'organigrammes, elles furent assimilées aux Ressources humaines, puis aux Communications, puis aux Affaires générales avant d'être reprises par les Approvisionnements et Services.

Avant 1963, les réalisateurs radio et télévision avaient tendance à conserver dans leurs bureaux les rubans et bobines de leurs propres émissions. Après un certain temps, le manque d'espace les obligea à songer à un lieu d'archivage bien organisé. En janvier 1964, le manuel de gestion présentait la politique de la Société Radio-Canada sur la conservation et l'aliénation des enregistrements de télévision sur films, ce que l'on appelait les cinégrammes. Le Service du film était mandaté pour appliquer cette politique, à savoir d'“assurer la conservation adéquate des émissions retenues et l'aliénation des autres"; dans ce dernier cas, l'approbation du directeur de la télévision était nécessaire ${ }^{1}$.

\section{Sélection pour archivage}

Cette politique énumère les catégories et critères de sélection des enregistrements télévision à des fins d'archivage.

\section{Quatre catégories sont retenues}

1. Sociologique: la télévision comme activité majeure dans la vie des Canadiens, venant en troisième lieu après le sommeil et le travail.
2. Historique: la télévision est l'actualité; non seulement illustre-t-elle l'histoire en montrant l'actualité, mais elle devient elle-même un événement historique.

3. Artistique: la télévision est un médium d'expression créatrice ; elle absorbe les meilleurs talents canadiens.

4. Référence: des émissions seront retenues à des fins de référence, d'étude et d'analyse. Ce qui place cette quatrième catégorie à part des trois autres.

Dans la catégorie sociologique, les émissions sont retenues selon une grille élaborée pour éviter l'arbitraire et les choix subjectifs.

\section{TABLEAU 1 \\ Grille de sélection \\ pour émissions de catégorie sociologique}

Fréquence des émissions

Quotidienne

Hebdomadaire (52/an)

Hebdomadaire (39/an)

Hebdomadaire (26/an)

Hebdomadaire (13/an)

Moins fréquentes

\section{Quantité retenue}

6 par an

4 par an

3 par an

2 par an

1 par an

1 par an
Dans la catégorie historique, sont conservées des émissions pour leur valeur permanente ou à long terme, reflétant l'histoire de la télédiffusion de Radio-Canada, la naissance, la vie et la mort des émissions; I'histoire canadienne reflétée par les événements contemporains antérieurs et les biographies; I'histoire mondiale selon les mêmes critères que précédemment mais considérés dans une optique internationale.

Dans la catégorie artistique, le choix se porte sur une production télévisuelle dont l'excellence des divers éléments la composant en fait un produit de grande valeur, justifiant l'attribution d'un prix national ou international.

La catégorie de référence regroupe des émissions qui pourront être éliminées plus tard, lorsqu'on en aura tiré tout l'enseignement que leur mise en chantier aura apporté à l'équipe de production ${ }^{2}$. 
Nous nous inspirons encore de cette politique de 1964 dans la sélection hebdomadaire des émissions à archiver, en consultant la grille horaire. Est-il besoin d'ajouter que la même philosophie prévaut pour les émissions de radio.

Une politique générale émise en 1978 au sujet de la conservation de bandes magnétoscopiques se lit ainsi :

La conservation des bandes magnétoscopiques est déterminée par les possibilités de rediffusion nationale ou régionale, d'échanges avec les organismes étrangers de télévision, de commercialisation, de dépôt à des services d'archives locales ou nationales, ou enfin par l'intérêt qu'elles peuvent avoir pour l'information et la formation professionnelles. Toute émission significative, ou exceptionnelle du point de vue artistique, culturel, religieux, scientifique, politique, sportif ou historique, doit être conservée par la Société.

Cette politique vise à éviter, d'une part, la destruction d'émissions remarquables et à empêcher, d'autre part, les abus en matière de conservation, lesquels entraîneraient des immobilisations coûteuses, des frais de locaux et de gestion injustifiables, en plus d'entraver la réutilisation des bandes magnétoscopiques ${ }^{3}$.

\section{Collaboration avec les Archives nationales du Canada}

Déjà en 1975, une entente avait été ratifiée avec les Archives nationales du Canada (ANC) selon laquelle les documents sonores et visuels de la Société étaient versés en priorité aux ANC, avant de l'être aux archives provinciales ou d'universités. Une mise à jour de cet accord fut signée en 1981, dans laquelle on peut lire entre autres:

\section{Soin des documents:}

Les Archives publiques exerceront un soin professionnel dans la garde des documents, conformément aux méthodes habituelles de préservation en ce qui a trait à la conservation de ces documents et à l'accessibilité des documents aux fins de recherche et d'étude, afin de les préserver contre la perte, les avaries et la détérioration.

Les Archives publiques ne céderont aucun document de Radio-Canada sans le consentement écrit de cette dernière.
Les Archives publiques créeront un index central de toutes les collections de documents de Radio-Canada qu'elles auront en leur possession et de toute collection de documents des Archives publiques directement liés à Radio-Canada.

Les Archives publiques mettront des exemplaires de cet index à la disposition de RadioCanada.

Les Archives publiques consulteront RadioCanada en ce qui concerne l'établissement de normes communes pour l'équipement ou pour les méthodes d'entretien de façon à garantir la plus haute qualité possible de la diffusion d'émissions.

Les émissions et éléments d'émissions désignés par Radio-Canada aux fins de conservation spéciale seront gardés par les Archives publiques et maintenus sur leur support original de façon qu'il n'y ait aucune dégradation de la qualité de ces émissions aux fins d'une diffusion ultérieure ${ }^{4}$.

C'est la loi sur les Archives nationales du Canada qui établit la «mission et attributions» de cet organisme:

4. (1) Les Archives nationales du Canada conservent les documents privés et publics d'importance nationale et en favorisent l'accès. Elles sont le dépositaire permanent des documents des institutions fédérales et des documents ministériels. Elles facilitent la gestion des documents des institutions fédérales et des documents ministériels et appuient les milieux des archives ${ }^{5}$.

Ainsi l'archiviste national doit acquérir des documents des organismes fédéraux... prendre toute mesure utile au classement, à la description, à la protection et à la restauration des documents, etc. Cette obligation a rendu possible une collaboration entre les ANC et la Société RadioCanada, par laquelle cette dernière dépose des émissions de radio et de télévision selon un calendrier établi par les archives. Ces dépôts restent la propriété de la Société qui peut les rappeler en tout temps pour diffusion. L'archiviste fédéral permet aux chercheurs l'accès aux documents sous réserve des restrictions imposées par le droit d'auteur et les différents contrats liant la Société et les artistes. 
Cette collaboration entre les Archives nationales et la Société vaut à la fois pour les émissions de langue française et celles de langue anglaise. En 1975, lors de la signature du premier contrat, on a d'abord déposé des émissions de prestige françaises et anglaises et des grandes émissions d'affaires publiques réalisées par la zone d'Ottawa. L'entente signée en 1981 donna la priorité à la conservation des émissions à Radio-Canada. Globalement, la société d'État diffuse 150000 heures par an, radio et télévision réunies. Parmi ces documents radiophoniques accumulés depuis 1936 et télévisuels depuis 1952, certains ne représentent aucun intérêt pour les Archives. Aussi il s'est avéré nécessaire de conclure des ententes avec les archives provinciales et certaines universités qui se montrèrent intéressées par des émissions produites en région.

II y a donc des dépôts dans les archives des provinces suivantes: Colombie-Britannique, Alberta, Saskatchewan, Québec, NouveauBrunswick, Nouvelle-Écosse, Terre-Neuve/ Labrador. Les organismes suivants reçoivent des émissions sélectionnées selon leurs domaines d'intérêts: Centre d'Études acadiennes à Moncton, Memorial University Folklore and Language Archives, Université du Québec à Montréal, Université Concordia, Université de Montréal, Université York, Thunder Bay Historical Museùm. Ces ententes de dépôt impliquent un protocole par lequel l'organisme s'engage à ne pas laisser sortir les émissions hors ses murs, à ne pas les reproduire et à permettre en tout temps à la Société de les rappeler pour les besoins de sa production.

Un projet d'histoire orale est réalisé par l'Institut des études canadiennes à l'Université Carleton. Il fut organisé en 1981 par les ANC, la Société Radio-Canada et le doyen de l'Institut, alors qu'on s'inquiétait de la perte du patrimoine culturel canadien si l'on ne consignait pas les souvenirs des femmes et des hommes associés à RadioCanada.

\section{Gestion de la collection}

Pour être conservées, les émissions doivent être une production ou une co-production de Radio-Canada. Elles doivent répondre aux critères énoncés au début de cet article ou encore être identifiées comme du matériel pouvant être vendu sur les marchés étrangers. La sélection des émissions à retenir se fait à partir de la grille horaire présentée avant le début de la saison. Un ajustement hebdomadaire est fait pour incorporer les «spéciales», les changements d'horaire, etc.

À chaque jour, arrivent les rubans et les vidéocassettes de la veille, auxquels est attribué un numéro composé des deux chiffres de l'année, du mois, du jour suivi d'un tiret et d'un numéro séquentiel, 890402-1, 890402-2. Des analystes de contenu écoutent ou visionnent ces documents pour noter sur un bordereau les sujets traités et les images en indiquant le minutage. On note également les personnalités en ondes ou celles dont on parle ainsi que le générique. Ces informations transcrites sur fiches serviront à bâtir les divers catalogues dont se serviront les agents de recherche et les clients. Le service répond d'abord aux demandes des gens à l'interne qu'ils soient de la production, clientèle majoritaire, ou de l'administration. Les demandes extérieures proviennent de chercheurs universitaires ou de compagnies qui veulent utiliser le matériel pour produire des films, de la télévision ou de la publicité. Lorsqu'il y a des droits à libérer, le client externe fait affaire avec les Entreprises Radio-Canada qui sont habilitées à vendre la production de la Société. Les clients de l'extérieur doivent assumer un coût de service horaire aux archives de $30,00 \$$. Les Entreprises RadioCanada pour leur part ont des prix de vente proportionnels à la longueur du document et à la complexité des droits à libérer.

Les utilisateurs de la collection disposent de deux salles d'écoute et de deux salles de visionnement. Si une copie est nécessaire, elle est faite sur place par un technicien affecté en permanence aux transferts et repiquages. Certains clients internes sont des usagers quotidiens des archives et exigent des délais très courts: en sortant d'une réunion de production à 10:30, ils demandent un extrait à diffuser en ondes aux nouvelles de midi. Si le ruban ou la vidéocassette se trouve sur les rayons, passe encore! Mais lorsque le document est entreposé aux Archives nationales dans leur dépôt de Ville Saint-Laurent, c'est la mise en branle d'un courrier ultra-rapide et la course contre la montre commence.

La diversité des demandes est telle que toutes les émissions conservées, quelle que soit leur catégorie, sont susceptibles d'être utilisées. Lorsque I'analyse et l'indexation sont faites, le repérage est rapide. Mais le traitement accuse des années d'arrérage et par conséquent, la seule identification est une feuille de route portant le titre et le numéro de support de l'émission. Au fur et à mesure que ces émissions seront déposées aux Archives nationales et traitées, des catalogues seront produits qui permettront une identification adéquate de ces trésors d'archives.

\section{Impact sur le milieu}

Même si la contribution quotidienne des archives d'émissions à la programmation radiophonique et télévisuelle n'est pour ainsi dire 
jamais soulignée au générique, nous savons que sans ce matériel bon nombre d'émissions n'auraient jamais pu être produites.

Les émissions de radio et de télévision de la Société sont parmi les documents les plus vitaux de l'histoire et de la culture canadiennes. Non seulement témoignent-elles d'événements mais, de plus en plus, elles constituent ellesmêmes des manifestations importantes, formant et conditionnant la perception qu'ont les Canadiens de leur société et du monde. Les émissions sont un héritage précieux qui doit être préservé pour la recherche et I'histoire. D'autre part, la collection et l'organisation de tous les documents, statistiques et rapports de Radio-Canada sont nécessaires aux historiens et aux universitaires qui cherchent à comprendre l'histoire et la culture de notre pays ${ }^{6}$.

\section{Michelle Bachand}

Responsable des archives d'émissions

Société Radio-Canada

Montréal

6. Société Radio-Canada, Services nationaux des programmes, 10 novembre 1986 (document interne). $\begin{array}{ll}\text { Statistics } & \text { Statistique } \\ \text { Canada } & \text { Canada }\end{array}$

\section{UN GRAND LIVRE À L'IMAGE D'UN GRAND PAYS}

Statistique Canada présente .....

\section{Un Portrait du Canada}

L'organisme statistique national a uni ses efforts à ceux de photographes canadiens réputés pour donner un portrait vivant et informatif du Canada d'aujourd'hui.

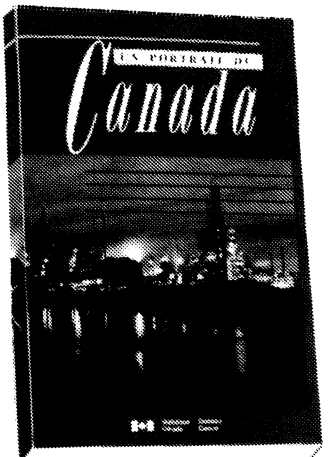

Illustré de plus de 200 photos couleur, de graphiques et de tableaux, Un Portrait du Canada présente une image détaillée des aspects suivants :

* Le territoire... l'environnement et le climat.

* Le peuple... son héritage et ses caractéristiques démographiques.

* La société... les arts, l'éducation, la santé et les loisirs.

* L'économie... la main-d'oeuvre, les branches d'activité et le commerce.

Pour un service plus rapide, appelez sans frais au 1-800-361-2831. 